\title{
eJRIEPS
}

Ejournal de la recherche sur l'intervention en éducation physique et sport

Numéro spécial 3 | 2020

Actes de la 11 ème Biennale de l'ARIS, Lille, 19-21 juin

2018

\section{Arbitrer en EPS : une expérience de responsabilisation}

Caroline Ganière, David Adé et Benoît Louvet

\section{(2) OpenEdition \\ Journals}

Édition électronique

URL : http://journals.openedition.org/ejrieps/4491

ISSN : 2105-0821

Éditeur

ELLIADD

\section{Référence électronique}

Caroline Ganière, David Adé et Benoît Louvet, «Arbitrer en EPS : une expérience de

responsabilisation », eJRIEPS [En ligne], Numéro spécial 3 | 2020, mis en ligne le 01 janvier 2020, consulté le 18 février 2020. URL : http://journals.openedition.org/ejrieps/4491

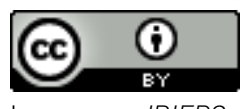

La revue eJRIEPS est mise à disposition selon les termes de la Creative Commons Attribution 4.0 International License. 


\section{Arbitrer en EPS : une expérience de responsabilisation}

Caroline Ganière*, David Adé* \& Benoît Louvet*

* Laboratoire CETAPS, EA 3832, Université de Rouen Normandie

Résumé

Cette étude s'intéresse à l'expérience d'arbitrage d'élèves dans des leçons d'EPS. Centrée sur des données d'expérience vécue, cette étude les enrichit avec des données relatives à la motivation des élèves dans le but de mettre à jour leurs stratégies pour occuper le rôle d'arbitre et expérimenter la responsabilité inhérente à ce rôle. Les données d'expérience ont été recueillies et traitées dans le cadre théorique et méthodologique du cours d'action (Theureau, 2004, 2006). Les données motivationnelles ont été recueillies et traitées dans le cadre théorique de la motivation auto-déterminée (Deci \& Ryan, 2002). Les résultats font apparaitre (1) trois préoccupations typiques (tenir le rôle d'arbitre, s'aider des autres, se délester du rôle d'arbitre), et (2) des formes d'amotivation, de régulation externe et de motivation intrinsèque à la connaissance et à l'accomplissement, ainsi qu'un besoin d'autonomie plus faible que ceux de compétence et d'affiliation. Sur cette base sont (1) discutés le repérage de stratégies d'alternance entre ces préoccupations typiques caractérisant chez les élèves leur façon d'assumer des responsabilités, et (2) proposées des aides à l'intervention pour concevoir des environnements encourageant les élèves à occuper le rôle d'arbitre à partir de gestalts.

Mots clés : Cours d'action, Expérience, Motivation, Arbitre, Rôle social, Education Physique et Sportive.

\section{Summary}

This research investigates students' refereeing experience in physical education (PE) classes. Focused on lived experience datas, this study enhances them with students' motivational datas in order to highlight their strategies to assume the role of referee and experiment with the specific responsabilities of this role. Experience datas were collected and processed in the theoretical framework of course-of-action (Theureau, 2004, 2006). Motivational datas were collected and processed in the theoretical framework of self- 


\section{eJRIEPS spécial 3 (janvier 2020)}

determination motivation (Deci \& Ryan, 2002). Results showed (1) three typical concerns (fulfilling the role, getting help, and occasionally dropping out of the role), and (2) that students mainly expressed amotivation, external regulation and intrinsic motivation toward knowledge and accomplishment, with a need of autonomy lower that their needs of affiliation and competence. On this basis, were (1) discussed the tracking of alternation strategies between these typical concerns characterizing how students assume specific responsibilities and (2) proposed aid to intervention to design environments encouraging students' to assume the role of referee from identification of gestalts.

Key words: Course-of-action, Experience, Motivation, Referee, Social role, Physical Education.

\section{Introduction}

Cet article s'intéresse à l'activité des élèves lorsqu'ils occupent le rôle d'arbitre au cours de leçons d'Education Physique et Sportive (EPS) en France. Ce rôle participe selon les références programmatiques à construire l'une des compétences travaillées en EPS, consistant à « partager des règles, assumer des rôles et des responsabilités » (MEN, 2015). Ainsi, au collège comme au lycée, les enseignants trouvent des occasions pour faire par exemple « observer et co-arbitrer » les élèves (MEN, 2015) ou " respecter des règles de vie collective et assumer les différents rôles liés à l'activité » tels que « juger, arbitrer, aider (...) » (MEN, 2010). Ces compétences restent présentes dans les nouveaux programmes du lycée général et technologique où il est question, entre autres, d'apprendre aux élèves à " assurer différents rôles (arbitre, coach, observateur ...) » et de "connaître et faire respecter les règles » (MEN, 2019). Ces prescriptions institutionnelles présupposent ainsi des liens forts entre l'expérience d'arbitrage et celle de la responsabilité. Toutefois ce concept de responsabilité est complexe puisqu'il s'agit à la fois de "prendre acte de l'incertitude quant à la pertinence de nos choix et de se confronter à l'obligation d'en assumer les conséquences " (Jaeger, 2009, p. 73). La responsabilité fait ainsi référence à la possibilité d'imputer une action à une personne ou de la lui attribuer en tant qu'elle en est le véritable auteur, comme le fait l'arbitre lorsqu'il repère une faute délibérément commise par un joueur sur un autre. Ainsi, la responsabilité engage l'acteur dans plusieurs dimensions : la liberté dans ses choix, la décision, l'action, l'évaluation et éventuellement la 


\section{eJRIEPS spécial 3 (janvier 2020)}

sanction (Jaeger, 2009). La responsabilité est bien plus qu'une assurance propre à soi qui donne la force d'assurer les conséquences des actes posés. En effet, prendre des responsabilités revient à estimer ses actes, leur(s) portée(s), leur(s) effet(s) et la relation à autrui (Janner-Raimondi, 2015). La responsabilité est donc intersubjective (Jardin, 2008) car elle invite "l'individu à s'occuper sans cesse du point de vue d'autrui pour le comparer au sien " (Piaget, 1932, p. 149), afin de prendre ses propres décisions et de les assumer. II semblerait donc qu'occuper le rôle d'arbitre dans une leçon d'EPS, soit à la fois une activité individuelle et collective.

L'objectif de cet article est d'étudier l'expérience vécue par les élèves en situation d'arbitrage afin de mettre à jour les stratégies qu'ils déploient pour y être efficaces de leur point de vue. Nous faisons l'hypothèse qu'en comprenant le rôle d'arbitre « du dedans " (Lerbet, 1992 ; Sève \& Terré, 2018), nous pourrons caractériser les façons qu'ont les élèves de faire face à une expérience de responsabilisation.

Le rôle d'arbitre se distingue d'autres rôles sociaux comme par exemple ceux d'observateur, de coach ou de pareur que les élèves sont amenés à découvrir en EPS. Il est non seulement indissociable de certaines pratiques physiques et sportives en EPS (e.g., les sports collectifs), implique une visibilité publique importante dans la classe et impacte aussi directement l'issue d'un match. Pour autant il reste à ce jour peu étudié (Adé, Ganière, \& Louvet, 2018). En effet, la plupart des études s'étant intéressées aux rôles sociaux en EPS se sont concentrées sur les rôles de coach ou de tuteur (Lafont, Rivière, Darnis, \& Legrain, 2016). Ces études ont notamment été menées en psychologie sociale dans le cadre des courants du cooperative learning (Lafont, 2012) et du peer assisted learning (Jenkison, Naughton, \& Benson, 2014). Elles ont permis de mettre en évidence les bénéfices du travail collaboratif de groupe et entre pairs (pour les élèves tuteurs et tutorés) pour des apprentissages moteurs, méthodologiques et sociaux et de pointer la plus-value apportée par des tuteurs préalablement formés et intervenant dans le cadre de dyades dissymétriques.

D'autres études ont également investigué les rôles sociaux pouvant être tenus par des élèves en EPS mais sous l'angle de l'expérience vécue. C'est le cas de celles conduites dans le programme de recherche du cours d'action (CA) (Saury et al., 2013 ; Theureau, 2002, 2006) dans lequel s'inscrit cette étude. Ce programme de recherche a montré sa fécondité dans l'analyse des situations d'enseignement de l'EPS notamment en ce qui concerne l'étude de l'activité des élèves en classe (pour une synthèse voir Saury et al., 2013). Ces études ont notamment mis en lumière l'émergence d'offres et de demandes 


\section{eJRIEPS spécial 3 (janvier 2020)}

d'aides spontanées entre élèves (e.g., Adé, Picard, \& Saury, 2013 ; Huet \& Saury, 2011 ; Saury \& Rossard, 2009), et les liens entre les interactions entre élèves et les caractéristiques spatiale, temporelle et matérielle de la leçon (e.g., Adé, 2016 ; Jourand, Adé, Sève, Komar, \& Thouvarecq, 2017).

Le programme de recherche du CA repose entre autres sur deux postulats spécifiant la notion d'activité à partir de l'expérience vécue par les acteurs : le postulat de l'énaction et celui de la conscience pré-réflexive.

L'énaction (Maturana \& Varela, 1994) traduit l'hypothèse de relations circulaires autoréférencées entre l'acteur et l'environnement. Ainsi, à chaque instant, l'activité ne traduit pas une adaptation à un environnement mais une spécification de ce qui, pour l'acteur est significatif dans cet environnement, c'est à dire le perturbe. Ces relations circulaires s'accompagnent de transformations conjointes de l'acteur et de l'environnement pour compenser les perturbations de l'environnement. Autrement dit, il s'agit ici de considérer que l'activité étudiée est celle d'un acteur qui « ne subit pas la force prescriptive des stimuli de l'environnement, mais recherche un état d'équilibre en sélectionnant ses propres perturbations » (Sève \& Saury, 2010). Par exemple, le signalement de fautes de jeu (comme un marcher en basket-ball) par des élèves en attente sur le bord du terrain de basket, va participer à définir le monde propre des élèves occupant le rôle d'arbitre : certains vont y voir des opportunités d'aide et d'autres des tentatives d'influences. L'activité fait donc émerger des « micro-mondes » (Varela, 1989) qui sont énactés à chaque instant. Cette idée est synthétisée en définissant l'acteur comme structurellement couplé avec son environnement.

Mais, du fait de l'hypothèse de l'énaction, ce couplage est asymétrique dans le sens où chaque acteur possède un point de vue propre sur son environnement. Tous les élèves arbitres ne perçoivent donc pas les mêmes éléments significatifs dans l'environnement. Par exemple, alors que pour certains élèves le signalement de fautes par des camarades spectateurs est perçu comme une aide pour arbitrer, ce même élément peut être occulté chez un autre élève arbitre, du fait par exemple d'un bon niveau de maitrise de l'APSA en tant que joueur de club et de sa volonté d'assumer la responsabilité de ses actes.

Dans ce couplage structurel asymétrique qui se transforme en permanence, l'acteur construit donc à chaque instant des significations définissant son monde propre. Pour rendre compte de ces significations, nous nous référons à l'objet théorique « cours d'expérience ». Le cours d'expérience est «la construction du sens pour l'acteur de son activité au fur et à mesure de celle-ci » (Theureau, 2006, p. 48). II correspond à une 


\section{eJRIEPS spécial 3 (janvier 2020)}

réduction de l'activité au niveau de ce qui en est mimable, montrable, racontable et commentable et constitue un accès pertinent à l'effet de surface de la dynamique du couplage structurel entre l'acteur et son environnement. C'est cette expérience partielle du couplage structurel entre l'acteur et l'environnement que Theureau $(2004,2006)$ désigne par « conscience pré-réflexive ". La conscience pré-réflexive est donc une composante de l'activité qui, moyennant certaines conditions méthodologiques (Theureau, 2004, 2006), donne accès à la partie que l'acteur peut montrer, commenter et raconter à un observateurinterlocuteur. Ainsi, un élève peut expliciter ce qu'il fait, cherche à faire, ressent, prend en compte lorsqu'il vit une expérience d'arbitrage et de responsabilité à travers ce rôle.

Si ces études menées dans le cadre du CA n'ont pas abordé spécifiquement le rôle social d'arbitre lorsqu'il est prescrit par un enseignant, c'est le métier d'arbitre qui a été bien plus investigué à ce jour dans le milieu sportif professionnel.

La littérature sur les officiels en sport a fait la part belle aux difficultés liées au rôle d'arbitre notamment sur les dimensions émotionnelles et décisionnelles (Louvet, 2011). Les recherches centrées sur les aspects émotionnels mettent en avant les conditions stressantes de cette fonction. II a été montré que la principale émotion ressentie par les arbitres est l'anxiété (Taylor \& Daniel, 1988) dans la mesure où ils font face à de nombreuses sources de stress. Taylor et Daniel (1988) ont identifié six sources de stress liées à l'activité d'arbitrage : les conflits interpersonnels, la peur de l'agression physique, les conflits avec les pairs, les pressions temporelles, le manque de reconnaissance de la fonction et la peur de l'échec. Le caractère stressant de l'activité d'arbitrage est donc avéré et pourrait participer à limiter l'engagement des élèves dans ce rôle.

L'arbitrage a également fait l'objet d'études sur l'activité de jugement et de prise de décisions (Dosseville, Laborde, \& Raab, 2011), actes synonymes de prises de responsabilités. Par exemple, les travaux de Rix en rugby (2005) mettent en évidence trois types de jugements. Le " jugement en acte ", qui consiste à laisser le jeu se dérouler même si une faute est commise, correspond à la règle de l'avantage. Le « jugement de fait » qui caractérise l'application stricte du règlement et consiste à siffler la faute dès qu'elle survient. Enfin, le « jugement délibéré » spécifié par une activité d'assistance lors d'un arrêt de jeu, avec des pairs ou de la vidéo, pour confirmer (ou pas) la présence d'une faute. D'autres études ont identifié des typologies d'arbitres. Garncarzyk (1995), par exemple, en définit six en handball en fonction du niveau d'expertise des arbitres : les "non-interventionnistes" (rejettent l'aspect répressif et sont attentifs à la gestion du rapport de force) ; les arbitres «pragmatiques» (sensibles à la sécurité des joueurs et à l'équité du match); les 


\section{eJRIEPS spécial 3 (janvier 2020)}

"théoriciens》 (focalisés sur les dimensions règlementaires); les "stressés" (qui pratiquent souvent par obligation pour être au service d'un club par exemple); les " autoritaires » (limitent les interactions avec les joueurs et s'appuient sur des sanctions disciplinaires) et enfin les «dilettantes» (se détournent ponctuellement des responsabilités).

Si ces études conduites dans le milieu professionnel sont utiles pour caractériser l'arbitrage et sur cette base influer la formation du corps arbitral (Rix-Lièvre, Boyer, Coutarel, \& Lièvre, 2014), elles se démarquent du contexte scolaire dans lequel la responsabilité de l'arbitrage est souvent prescrite par l'enseignant à des élèves qui s'y engagent rarement spontanément.

En effet, les élèves lui réservent parfois un accueil contrasté dans la mesure où ce rôle véhicule une réputation peu valorisante. L'arbitre est généralement perçu comme celui qui sanctionne, stimule la critique et les comportements transgressifs (Louvet, 2011). Nous faisons ainsi l'hypothèse que dans les situations où l'enseignant impose aux élèves d'arbitrer, ces derniers pourraient être faiblement motivés pour occuper ce rôle. Cette faible motivation pourrait être liée à la visibilité publique du rôle et aux responsabilités qu'il cristallise.

II nous a donc paru intéressant d'éclairer les données d'expérience avec des données concernant la motivation des élèves dans l'objectif de mieux comprendre les stratégies mises en place lors de l'activité d'arbitrage. Ces données relatives à la motivation sont adossées à la théorie de l'autodétermination (Deci \& Ryan, 2002). Cette théorie envisage trois formes principales de motivation: l'amotivation, la motivation extrinsèque et la motivation intrinsèque. Un élève amotivé ne perçoit aucun lien entre ses actions et les résultats qu'il obtient. S'il est motivé extrinsèquement, l'élève s'engage dans une activité pour les conséquences qu'elle peut lui procurer soit (1) pour obtenir une récompense ou éviter une punition (régulation externe, (2) en raison d'un sentiment de culpabilité (régulation introjectée) ou (3) pour réaliser une activité en cohérence avec ses propres valeurs (régulation intégrée). Les régulations introjectée et intégrée ont un degré d'autodétermination supérieur à la régulation externe et sont donc plus favorables à l'implication de l'individu dans les situations. Enfin, un élève de plus en plus autodéterminé sera motivé intrinsèquement ; il s'engagera librement dans une activité pour le plaisir qu'elle lui procure (motivation intrinsèque à la stimulation), pour le développement de ses capacités (motivation intrinsèque à l'accomplissement) ou encore pour les opportunités d'apprendre qu'elle lui offre (motivation intrinsèque à la connaissance). Ces différentes régulations 


\section{eJRIEPS spécial 3 (janvier 2020)}

motivationnelles sont déterminées par la satisfaction de trois besoins psychologiques fondamentaux. II s'agit du besoin d'autonomie (se sentir être à l'origine de ses propres actions), du besoin d'affiliation (se sentir connecté aux autres) et du besoin de compétence (se sentir efficace).

Au total cette étude est porteuse d'un double enjeu. Un premier enjeu de nature épistémique vise l'élaboration de connaissances relatives à l'expérience d'arbitrage des élèves ainsi qu'aux caractéristiques motivationnelles associées à cette expérience. Un second enjeu, transformatif, vise à proposer des pistes d'intervention afin de stimuler l'engagement des élèves dans ce rôle à des fins de développement d'expériences de responsabilités.

\section{Méthode}

\subsection{Participants à l'étude et situations étudiées}

Les situations étudiées étaient des matchs de sports collectifs (basket-ball, futsal et handball) d'une durée de 8 minutes et se déroulant à la fin des leçons numéro 3,5 et 7 sur des séquences de 8 leçons avec des classes de quatrième (élèves de 13-14 ans). A ces occasions, l'enseignant avait demandé aux élèves arbitres de porter attention aux règles relatives à la marque, la conduite du ballon (règles du marcher et de la reprise de dribble) et à sa conservation dans l'aire de jeu (touches).

74 élèves (35 garçons et 39 filles) issus de trois classes différentes ont participé à l'étude. Parmi eux, 4 élèves (2 filles et 2 garçons) ont été volontaires pour être filmés lorsqu'ils arbitraient et pour participer à des entretiens post-matchs. Ces élèves n'étaient pas experts des activités sportives dans lesquelles ils arbitraient et étaient reconnus comme de bons élèves en EPS, parfaitement intégrés dans le groupe classe. Ces élèves n'ont pas renseigné les questionnaires relatifs à la motivation contrairement à tous les autres élèves.

\subsection{Recueil des données}

Deux types de données ont été recueillis: des données d'expérience et des données relatives à la motivation. Les données d'expérience constituent pour cette étude une entrée prioritaire mais non exclusive. Du fait de leur faible niveau d'engagement supposé pour occuper le rôle d'arbitre, nous avons enrichi ces données d'expérience par des données relatives à la motivation des élèves.

\subsubsection{Recueil des données d'expérience}

Dans un premier temps, des enregistrements audio-visuels de l'activité d'arbitrage ont été effectués en filmant en continu et en plan large à l'aide d'une caméra équipée d'un micro 


\section{eJRIEPS spécial 3 (janvier 2020)}

haute fréquence. 106 minutes d'enregistrement d'activité d'arbitrage ont été recueillies. Ces données d'enregistrement ont servi de support à des entretiens d'autoconfrontation individuels conduits immédiatement après chaque match. Cette procédure consiste à confronter chaque élève à l'enregistrement de son activité pour l'encourager à expliciter, montrer et commenter les éléments significatifs pour lui de cette activité, en présence du chercheur (Theureau, 2004, 2006). Elle visait à recueillir des données permettant de documenter le cours d'expérience des élèves. Les entretiens, d'une durée maximale de trente minutes compatible avec la disponibilité des élèves, ont représenté un corpus de plus de 400 minutes d'enregistrement.

\subsubsection{Recueil des données relatives à la motivation}

Ces données ont été recueillies par le biais de deux questionnaires distribués aux élèves juste après leur expérience d'arbitrage.

Le questionnaire IMI : Intrinsic Motivation Inventory (McAuley, Duncan \& Tammen, 1989 ; Cury, Famose \& Sarrazin, 1994, en français) a été utilisé pour mesurer les besoins psychologiques fondamentaux à l'origine de la motivation auto-déterminée (Vallerand, 1997). II comportait quinze items permettant de mesurer respectivement : la compétence perçue (cinq items dont : " je ne me sens pas très compétent »), le besoin d'affiliation (cinq items dont : "je considère les personnes avec qui je fais cet exercice comme des amis") et le besoin d'autonomie (cinq items dont : " je me sens libre de mes choix »). Ces quinze items étaient évalués sur une échelle de type Likert allant de 1 (pour "Pas du tout vrai ») à 7 (pour « complètement vrai »). Les Coefficients alpha de Cronbach pour chaque besoin allaient de $.75, .78$, et .85 , et révèlent une très bonne consistance interne.

Le second questionnaire, précédemment utilisé par Boiché, Sarazin, Grouzet, Pelletier, et Chanal (2008) en EPS, a permis d'évaluer les régulations motivationnelles : la motivation intrinsèque à la connaissance ou à l'accomplissement ("pour le plaisir que je ressens lorsque j'améliore certaines de mes faiblesses »), la motivation intrinsèque à la stimulation (" pour l'excitation, pour les sensations que me procure cette matière "), la motivation extrinsèque identifiée (" parce que ce que j'apprends dans cette matière me sera utile plus tard "), la motivation extrinsèque introjectée ("parce que j'ai absolument besoin de réussir dans cette matière "), la motivation extrinsèque de type régulation externe ("parce que je ne veux pas décevoir des gens importants ") et l'amotivation ("Je ne sais pas pourquoi je viens en EPS»). Ce questionnaire se compose de dix-huit items accompagnés d'une échelle de type Likert en sept points allant de "totalement en désaccord » à « totalement en accord ». Les Coefficients alpha de Cronbach des différentes 


\section{eJRIEPS spécial 3 (janvier 2020)}

sous-échelles de régulation motivationnelle se situent entre .75 et .85 et attestent d'une très bonne consistance interne.

\subsection{Traitement des données}

\subsubsection{Traitement des données d'expérience}

Les données d'expérience ont été traitées en deux étapes : (1) la reconstruction du cours d'expérience de chacun des quatre élèves arbitres ; (2) l'identification de préoccupations typiques.

\subsubsection{La reconstruction du cours d'expérience}

La reconstruction des cours d'expérience a consisté pour chaque élève à identifier les unités significatives pour eux et émergeant de leur interaction avec leur environnement, ainsi que les composantes de chacune d'elles. Les trois composantes qui ont été considérées dans le cadre de cette étude sont l'unité significative élémentaire, le representamen, et l'engagement dans la situation. La documentation de ces composantes a été conduite grâce à une analyse simultanée des enregistrements audio et vidéo des leçons, des retranscriptions verbatim des verbalisations des entretiens d'autoconfrontation, et d'un questionnement spécifique pour chacune d'elles.

L'unité significative élémentaire $(U)$ est la fraction de l'activité qui est montrée, racontée et commentée par l'acteur. Elle peut être une interprétation, un sentiment, une action ou une communication (e.g., "Siffle en fixant du regard le porteur de balle "). Elle a été identifiée par un questionnement du type : Que fait l'élève ? Que pense-t-il ? Que ressent- t-il ? Le representamen $(R)$ est ce qui, à l'instant $t$, fait signe pour l'acteur. II peut être un jugement perceptif, mnémonique ou proprioceptif (e.g., « les remarques d'élèves spectateurs »). II a été identifié par un questionnement du type : Que prend en compte l'élève ? Quel est l'élément perçu, rappelé ou interprété, qui est significatif pour lui ? L'engagement (E) dans la situation exprime les préoccupations saillantes en fonction de ce qui fait signe pour l'acteur (i.e. le (R)) à un instant précis (e.g., « rechercher l'approbation de sa décision auprès de l'enseignant») et qui est actualisé par l'acteur (i.e. le (U)). Il a été identifié par un questionnement du type : Quelles sont les préoccupations de l'élève en fonction des éléments pris en compte dans la situation ? Les Tableaux I à VI illustrent par des extraits la reconstruction du cours d'expérience d'élèves arbitre.

Sur cette base, nous avons caractérisé les préoccupations typiques pour identifier chez les élèves arbitres des formes similaires d'engagement. 


\subsubsection{L'identification de préoccupations typiques}

L'identification du caractère de typicité des préoccupations a été réalisée en référence à quatre aspects (descriptif, statistique, génératif et significatif) convoqués pour identifier des occurrences-types (Durand, 2014). Ainsi, des préoccupations sont qualifiées de typiques quand (1) elles concentrent le plus d'attributs de l'activité observée au sein de l'échantillon des acteurs et des situations étudiées ; (2) elles sont les plus fréquemment observées dans l'échantillon enquêté ; (3) elles ont une propension à s'actualiser de façon privilégiée lorsque des conditions ayant un air de famille avec celles observées se reproduisent ; (4) elles sont l'objet d'un sentiment de typicité exprimé par les élèves durant leurs interactions avec les chercheurs.

\subsubsection{Traitement des données quantitatives}

Les comparaisons entre les différentes régulations motivationnelles et entre les besoins psychologiques fondamentaux ont été effectuées avec une ANOVA à mesures répétées suivies d'une série de tests post-hoc (Least Significant Difference) pour effectuer des comparaisons deux à deux. Le seuil de significativité retenu a été de .05 .

\section{Résultats}

Nous présenterons deux lignes de résultats. La première est relative à l'expérience des élèves arbitres à partir de leurs préoccupations typiques. La deuxième ligne de résultats cible la motivation des élèves à occuper le rôle d'arbitre.

\subsection{L'expérience des élèves arbitres}

L'analyse et la comparaison des cours d'expérience des élèves arbitres ont permis de mettre en évidence la présence durant les situations de match de trois préoccupations typiques : tenir le rôle d'arbitre; s'aider des autres et se délester ponctuellement des fonctions du rôle. Nous présentons successivement les caractéristiques de chacune de ces préoccupations typiques en précisant les actions et focalisations auxquelles elles sont associées dans l'expérience des élèves.

\subsubsection{Tenir le rôle d'arbitre}

Cette préoccupation émerge dès lors que l'enseignant désigne un élève pour être arbitre. Dans ce cas, les élèves se sentent engagés dans les responsabilités qu'ils associent à ce 


\section{eJRIEPS spécial 3 (janvier 2020)}

rôle, ce qui les amène à se remémorer les règles du jeu pour répondre à la commande de l'enseignant et éviter de commettre des erreurs. Ces erreurs sont pour les élèves des sources potentielles de désaccords avec les joueurs susceptibles de remettre en question leur compétence à occuper le rôle (cf. Tableau I).

Tableau I : extrait du cours d'expérience de Martin

\section{Extrait autoconfrontation:}

Chercheur : " Donc ça y est, l'enseignante te désigne. À quoi tu penses à ce momentlà ? »

Martin: "Bah je me dis que c'est parti et j'essaye de me rappeler des règles d'arbitrage, comment il faut arbitrer... »

Chercheur : "Comment il faut arbitrer...? »

Martin : "Bah oui vu que je ne connais pas toutes les règles, j'essaye de me rappeler celles que le prof m'a demandé de siffler et celles dont je me souviens pour essayer de bien faire parce que c'est un peu particulier pour moi... ”

Chercheur : "Un peu particulier?»

Martin : "Oui parce que s'il y en a un qui fait une faute et qu'au final je ne la siffle pas, bah ça va se retourner contre moi, l'arbitre du match. Donc je me dis qu'il faut que j'arbitre bien du coup. ”

\begin{tabular}{|c|c|c|}
\hline U & R & $\mathrm{E}$ \\
\hline $\begin{array}{c}\text { Se lève du banc et se } \\
\text { dirige vers l'enseignant }\end{array}$ & $\begin{array}{c}\text { La désignation par } \\
\text { l'enseignant de l'élève } \\
\text { arbitre }\end{array}$ & $\begin{array}{c}\text { Se rappeler les règles du } \\
\text { jeu à prendre en compte } \\
\text { pour le match. } \\
\text { Remplir correctement sa } \\
\text { fonction }\end{array}$ \\
\hline $\begin{array}{c}\text { Se dirige vers le centre du } \\
\text { terrain }\end{array}$ & Le statut d'arbitre & $\begin{array}{c}\text { Répondre aux attentes de } \\
\text { l'enseignant. } \\
\text { Eviter de se mettre à dos } \\
\text { des joueurs }\end{array}$ \\
\hline
\end{tabular}

Une fois engagé dans le match, cette préoccupation se concrétise par le fait de siffler dès qu'il perçoit une faute par rapport au cadre déterminé par l'enseignant (un marcher, une reprise de drible) ou dès qu'un but est marqué. Pour cela, certains élèves arbitres cherchent à rester proches du porteur de balle et à garder un contrôle visuel sur celle-ci. Ce placement 


\section{eJRIEPS spécial 3 (janvier 2020)}

leur permet d'être efficaces de leur point de vue. Il les aide à mieux repérer les fautes, bien que cela nuise parfois au déroulement du jeu comme l'évoque Nina à qui il est arrivé d'être confondue avec un joueur partenaire et d'entrer en contact involontairement avec le ballon. (cf. Tableau II).

Tableau II : Extrait du cours d'expérience de Nina

\begin{tabular}{|c|c|c|}
\hline \\
\hline \multicolumn{3}{|c|}{ Nina : « Je cherche à toujours voir le ballon. » } \\
\hline \multicolumn{3}{|c|}{ Chercheur : «Et pour voir continuellement le ballon tu fais quoi ? » } \\
\hline \multirow{2}{*}{\multicolumn{3}{|c|}{$\begin{array}{l}\text { Nina : «Bah je reste à côté de celui qui dribble. Je me mets toujours à côté. J'aime } \\
\text { bien voir le ballon comme ça je vois mieux s'il y a des fautes. » }\end{array}$}} \\
\hline & & \\
\hline \multicolumn{3}{|c|}{ Chercheur : «Donc tu restes à côté pour mieux arbitrer. » } \\
\hline \multirow{2}{*}{\multicolumn{3}{|c|}{$\begin{array}{l}\text { Nina : « Oui même parfois on me fait la passe [pointe le doigt sur l'écran en rigolant]! } \\
\text { On me prend pour une joueuse! » }\end{array}$}} \\
\hline & & \\
\hline \multicolumn{3}{|l|}{$\mathrm{U}$} \\
\hline \multicolumn{3}{|l|}{$\begin{array}{l}\text { Court à côté du porteur de } \\
\text { balle }\end{array}$} \\
\hline $\begin{array}{l}\text { Se place à côté du porteur } \\
\text { de balle }\end{array}$ & $\begin{array}{l}\text { Le porteur de balle arrêté, } \\
\text { à la recherche d'un } \\
\text { partenaire }\end{array}$ & Rester près du ballon \\
\hline $\begin{array}{l}\text { Esquive le ballon et } \\
\text { rappelle au porteur de } \\
\text { balle qu'elle est arbitre }\end{array}$ & $\begin{array}{l}\text { Le ballon qui lui est } \\
\text { adressé }\end{array}$ & $\begin{array}{c}\text { S'excuser auprès des } \\
\text { joueurs d'avoir gêné le jeu } \\
\text { Rappeler qu'elle est } \\
\text { arbitre }\end{array}$ \\
\hline
\end{tabular}

D'autres élèves, au contraire, sont peu mobiles voire statiques. Dans ce cas, soit ils tiennent leur rôle en restant dans la zone centrale du terrain, soit ils se positionnent à la périphérie du terrain et à proximité de l'enseignant et/ou d'élèves spectateurs. Ce placement leur permet de demander ou saisir de l'aide auprès d'autres élèves et/ou de l'enseignant. Ils actualisent ainsi une autre préoccupation typique : « s'aider des autres ».

\subsubsection{S'aider des autres}

Lorsqu'ils s'aident des autres, les élèves arbitres cherchent à ne pas prendre seuls toutes les décisions. En effet, lors de moments de désaccords avec des joueurs, d'hésitation, ou 


\section{eJRIEPS spécial 3 (janvier 2020)}

encore lorsqu'une faute leur échappe, ils recherchent de l'aide pour confirmer ou infirmer la décision de signifier cette faute. Ils sont alors parfois sensibles aux réactions de certains joueurs qu'ils estiment plus compétents qu'eux dans la discipline. C'est par exemple le cas de Jules qui, lors d'une hésitation à signaler un « marcher », a attendu avant de siffler la confirmation par Gauthier reconnu en tant que joueur de club (cf. Tableau III).

Tableau III : Extrait du cours d'expérience de Jules

\section{Extrait autoconfrontation:}

Jules : " En fait j'ai eu un gros doute car je ne savais pas s'il avait vraiment marcher ou s'il changeait de direction. Du coup je n'ai pas sifflé de suite. Mais Gautier m'a dit qu'il y avait vraiment marcher "

Chercheur : "Tu as entendu Gautier (joueur dans la situation de match) te dire qu'il y a avait marcher? »

Jules : "Oui Gautier m'a fait la réflexion. Parce que lui il fait du handball en club et il connaît bien les règles. »

Chercheur : "Donc tu siffles "marcher » même si tu n'es pas sûr ?"

Jules : "Oui, mais c'est sûr il y avait « marcher » donc je siffle »

\begin{tabular}{|c|c|c|}
\hline U & R & E \\
\hline $\begin{array}{c}\text { Porte le sifflet à sa bouche } \\
\text { sans siffler }\end{array}$ & $\begin{array}{c}\text { Le déplacement du } \\
\text { porteur de balle }\end{array}$ & $\begin{array}{c}\text { Estimer si le joueur } \\
\text { commet ou pas un } \\
\text { «marcher » }\end{array}$ \\
\hline $\begin{array}{c}\text { Retire de sa bouche le } \\
\text { sifflet }\end{array}$ & $\begin{array}{c}\text { La difficulté à reconnaître } \\
\text { un « marcher » }\end{array}$ & $\begin{array}{c}\text { S'abstenir de siffler dans } \\
\text { le doute }\end{array}$ \\
\hline $\begin{array}{c}\text { Entends Gautier } \\
\text { l'interpeller }\end{array}$ & $\begin{array}{c}\text { L'annonce de Gautier qui } \\
\text { signale le « marcher » }\end{array}$ & $\begin{array}{c}\text { Se référer à l'évaluation } \\
\text { de Gautier }\end{array}$ \\
\hline Siffle & $\begin{array}{c}\text { La reconnaissance par } \\
\text { Gautier du « marcher » } \\
\text { Gautier est un joueur de } \\
\text { club }\end{array}$ & Signaler la faute \\
\hline
\end{tabular}

Dans d'autres cas, l'élève arbitre est sensible aux interventions de pairs signalant de loin une faute de jeu. Parfois même il interroge verbalement ou du regard l'enseignant, un élève joueur ou spectateur, pour estimer la convergence ou divergence de leurs réactions avec la décision prise dans le match. La décision de siffler est alors suspendue aux réactions de 


\section{eJRIEPS spécial 3 (janvier 2020)}

tierces personnes les incitant ainsi à ponctuellement « se délester du rôle d'arbitre » (cf. Tableau IV).

Tableau IV : Extrait du cours d'expérience de Lucie

\section{Extrait autoconfrontation :}

Chercheur : "Là on voit tu te retournes vers le banc? 》

Lucie : "Oui j’ai entendu des garçons crier « faute! ». »

Chercheur : "Et toi tu l'as vu la faute?»

Lucie : «Bah en fait je ne sais pas trop mais ils (des élèves assis sur le banc) crient « reprise de dribble ». »

Chercheur : "Donc tu les regardes?»

Lucie : "Oui et aussi la prof. Elle (la professeure) me fait un signe de la tête et puis les autres ils disent "madame il y a reprise ! ". Donc je siffle même si c'est un peu tard. »

\begin{tabular}{|c|c|c|}
\hline U & R & E \\
\hline vers le banc. & $\begin{array}{c}\text { Des garçons qui signalent } \\
\text { une reprise de dribble. }\end{array}$ & $\begin{array}{r}\text { Estimer la validité de } \\
\text { l'intervention des garçons }\end{array}$ \\
\hline Regarde l'enseignante & $\begin{array}{c}\text { Le sentiment d'avoir } \\
\text { oublié de signaler une } \\
\text { faute }\end{array}$ & $\begin{array}{c}\text { Avoir de l'aide de } \\
\text { l'enseignante }\end{array}$ \\
\hline Siffle la reprise de dribble & $\begin{array}{c}\text { Le signalement de la faute } \\
\text { par d'autres élèves } \\
\text { La confirmation par } \\
\text { l'enseignante }\end{array}$ & $\begin{array}{c}\text { Revenir sur la faute et la } \\
\text { siffler }\end{array}$ \\
& & \\
\hline
\end{tabular}

\subsubsection{Se délester du rôle d'arbitre}

Le rôle d'arbitre est vécu comme une tâche complexe et à forte responsabilité pour les élèves. Afin de faire face à la charge informationnelle, l'élève arbitre recherche ponctuellement à se décharger des responsabilités qui lui sont assignées. II profite ainsi de certaines occasions pour se distraire en rigolant avec des camarades ou pour se mettre en retrait en laissant s'installer ponctuellement des moments d'auto-arbitrage chez ses camarades joueurs.

Ces lâchers prise apparaissent lors de moments clés comme les phases de remise en jeu lors de touches ou à l'issue d'un but marqué. Ces moments sont pour l'élève arbitre, des 


\section{eJRIEPS spécial 3 (janvier 2020)}

opportunités pour s'évader de son rôle en actualisant des préoccupations orientées vers la distraction de façon à tenter de réduire le caractère anxiogène du rôle d'arbitre (cf. Tableau V).

Tableau V : Extrait du cours d'expérience de Lucie

\section{Extrait autoconfrontation:}

Chercheur : « Comment tu te sens à ce moment-là ? »

Lucie : "Je ne me sens pas encore tout à fait à l'aise... »

Chercheur : "D'accord. Et dans ces cas-là tu penses à quoi ? qu'est-ce que tu fais ? ” Lucie : «Bah là je rigole avec mes copains parce qu'ils n'arrêtaient pas de m'appeler. Chercheur : Et ils t'appellent souvent comme ça pendant le match ? »

Lucie: "Non mais ça m'arrive de discuter avec d'autres copains ou copines et de rigoler un peu. »

Chercheur : «Et vous discutez du match?»

Lucie: «Pas tout le temps [rigole], non...Et dès fois ils se moquent de moi. Mais comme ça je pense à autre chose. C'est bien [rigole]! »

\begin{tabular}{|c|c|c|}
\hline U & $\mathrm{R}$ & $E$ \\
\hline $\begin{array}{l}\text { Avec un sentiment de } \\
\text { stress, s'arrête et se } \\
\text { tourne en direction d'une } \\
\text { équipe en attente de jouer }\end{array}$ & $\begin{array}{l}\text { La présence de } \\
\text { camarades sur le bord du } \\
\text { terrain }\end{array}$ & Chercher du réconfort \\
\hline $\begin{array}{l}\text { Rigole avec ses } \\
\text { camarades }\end{array}$ & $\begin{array}{l}\text { Une plaisanterie d'un des } \\
\text { camarades }\end{array}$ & Penser à autre chose \\
\hline $\begin{array}{l}\text { Se rapproche de ses } \\
\text { camarades }\end{array}$ & $\begin{array}{l}\text { La remise en jeu à l'issue } \\
\text { d'un but offre un temps de } \\
\text { répit } \\
\text { La plaisanterie d'un des } \\
\text { camarades }\end{array}$ & Se détendre \\
\hline
\end{tabular}

À d'autres moments, l'élève arbitre se laisse parfois dépasser par le déroulement du match. II perd alors ponctuellement la main sur l'arbitrage sans que cela nuise au bon déroulement du match, du fait d'initiatives d'auto-arbitrage des joueurs. Ces moments sont alors vécus 


\section{eJRIEPS spécial 3 (janvier 2020)}

comme des occasions de ne plus assumer seuls les responsabilités inhérentes au rôle d'arbitre et d'atténuer le caractère anxiogène de la situation (cf. Tableau VI).

Tableau VI : Extrait du cours d'expérience de Martin

\section{Extrait autoconfrontation :}

Chercheur : "Là on t'entend siffler mais c'est après la remise en jeu ? 》 Martin: "Parce que la balle est sortie, mais en fait je n'ai même pas eu le temps d'indiquer pour quelle équipe c'était parce que le jeu avait déjà repris... » Chercheur : "Le jeu avait déjà repris ? »

Martin : "Oui, ils s'arbitrent un peu seuls pour des petites règles comme ça, je leur fais confiance. Mais après je siffle quand même. "

Chercheur : "Même en retard tu siffles ?»

Martin : "Bah oui sur le coup ils ont géré seuls, ça m'a aidé puis je leur dis c'est bon pour moi. Personne a râlé. Tout le monde était d'accord donc c'est bien comme ça. ”

\begin{tabular}{|c|c|c|}
\hline U & $\mathrm{R}$ & $E$ \\
\hline $\begin{array}{c}\text { Regarde la remise en jeu } \\
\text { sans réagir }\end{array}$ & $\begin{array}{l}\text { La remise en jeu de la } \\
\text { touche } \\
\text { L'absence de contestation }\end{array}$ & Laisser faire \\
\hline $\begin{array}{l}\text { Se rend compte que la } \\
\text { remise en jeu est } \\
\text { effectuée sans avoir eu le } \\
\text { temps de la siffler }\end{array}$ & $\begin{array}{l}\text { Le sentiment d'être en } \\
\text { retard } \\
\text { Le souvenir de ne pas } \\
\text { avoir vu la balle sortir du } \\
\text { terrain }\end{array}$ & $\begin{array}{l}\text { Laisser faire } \\
\text { Avoir confiance en les } \\
\text { joueurs }\end{array}$ \\
\hline Siffle en retard & $\begin{array}{l}\text { Un joueur qui vient de } \\
\text { faire la touche }\end{array}$ & Confirmer la remise en jeu \\
\hline $\begin{array}{l}\text { Se replace rassuré près } \\
\text { du porteur de balle }\end{array}$ & $\begin{array}{l}\text { Le souvenir d'avoir eu un } \\
\text { moment d'égarement } \\
\text { Le souvenir que dans ce } \\
\text { moment tout s'est bien } \\
\text { passé }\end{array}$ & $\begin{array}{c}\text { Reprendre la main sur } \\
\text { l'arbitrage }\end{array}$ \\
\hline
\end{tabular}




\section{eJRIEPS spécial 3 (janvier 2020)}

\subsection{La motivation des élèves arbitres}

Les moyennes et écart-types pour les différentes régulations motivationnelles et les besoins psychologiques fondamentaux sont présentés dans le tableau VII, ainsi que les corrélations entre les différentes variables.

Tableau VII : Moyennes et écart-types des régulations motivationnelles et des besoins psychologiques fondamentaux

\begin{tabular}{llllllllllll}
\hline Variables & $M$ & SD & 1 & 2 & 3 & 4 & 5 & 6 & 7 & 8 & 9
\end{tabular}

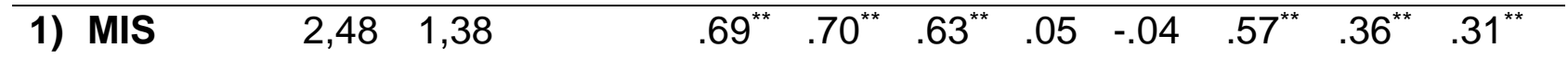

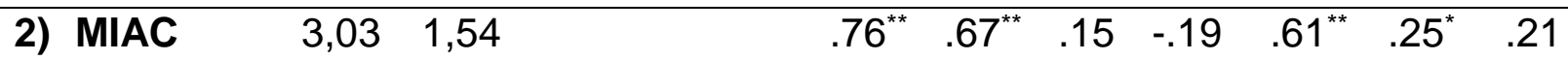

$\begin{array}{lllllllll}\text { 3) Rég. } & 2,49 & 1,60 & .74^{* *} & .17 & -.07 & .59^{* *} & .20 & .24^{*}\end{array}$
Iden.

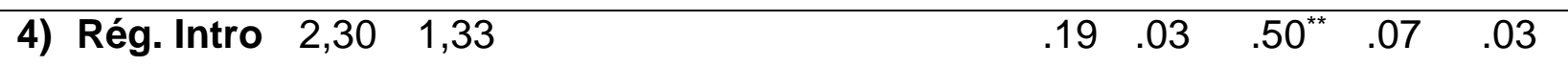

5) Rég. Ext. 2,98 1,47 $\quad \begin{array}{lllll} & .49^{* *} & -.08 & -.17 & -.44^{* \star}\end{array}$

$\begin{array}{llllll}\text { 6) Amot. } & 2,69 & 1,71 & -.06 & -.11 & -.36^{* *}\end{array}$

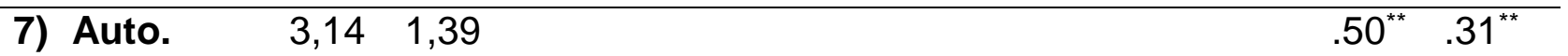

$\begin{array}{llll}\text { 8) Affil. } & 4,30 & 1,52 & .36^{* *}\end{array}$

\section{9) Comp. 4,37 1,07}

\footnotetext{
${ }^{* *} p<.01 . \quad{ }^{*} p<.05$

MIS : Motivation intrinsèque à la stimulation, MIAC: Motivation intrinsèque à la connaissance et à l'accomplissement, Rég. Ident.: Régulation identifiée, Rég. Intro. : Régulation introjectée, Rég. Ext. : Régulation externe, Amot : Amotivation, Auto. : Besoin d'autonomie, Affil. : Besoin d'affiliation, Comp. : Besoin de compétence
}

L'analyse de variance à mesures répétées révèle que les régulations motivationnelles sont ressenties à des niveaux significativement différents, $F(5,365)=4,05, p<.05$. En effet, les niveaux de régulations externes sont plus élevés que les degrés de motivation intrinsèque à la stimulation, de régulation identifiée et de régulation introjectée. Il est également à noter que la motivation intrinsèque à la connaissance et à l'accomplissement est plus importante que la motivation intrinsèque à la stimulation, la régulation identifiée, la régulation introjectée 


\section{eJRIEPS spécial 3 (janvier 2020)}

et l'amotivation. L'ensemble de ces régulations motivationnelles sont ressenties à des niveaux modérément faibles.

De plus, les résultats montrent que, lorsqu'ils arbitrent, les élèves ressentent des niveaux modérés d'autonomie, d'affiliation et de compétence (tableau VII). Ces déterminants motivationnels sont significativement éprouvés à des niveaux différents, $F(2,146)=32,57$, $p<.05$. Plus précisément, en situation d'arbitrage, le besoin d'autonomie des élèves est significativement plus faible que le besoin d'affiliation et que le sentiment de compétence. Ces derniers sont, quant à eux, ressentis à des niveaux similaires.

\section{Discussion}

Deux points de discussion sont développés. Le premier concerne l'identification de stratégies d'alternance chez les élèves pour faire l'expérience de la responsabilité lors de situations d'arbitrage. Le second vise à proposer des stratégies d'intervention chez les enseignants d'EPS à partir de l'identification de gestalts, c'est-à-dire des formes au sens de structures dynamiques et signifiantes pour les acteurs (Rosenthal \& Visetti, 1999).

\subsection{Des stratégies d'alternance chez les élèves pour faire l'expérience de la} responsabilité lors de situations d'arbitrage

L'analyse des expériences vécues a permis d'identifier deux stratégies d'alternance permettant aux élèves arbitres d'être efficaces de leur point de vue : une première alternance entre « assumer le rôle d'arbitre et s'en détacher » et une seconde entre " tenir le rôle d'arbitre seul et s'aider des autres ». Ces stratégies d'alternance rappellent l'identification du caractère circulaire de l'activité des élèves pointé par des recherches ancrées dans le paradigme de l'écologie de la classe (Allen, 1986 ; Hastie \& Siedentop, $1999,2006)$ et plus récemment par celles conduites dans le cadre du programme de recherche du CA (Saury et al., 2013 ; Vors \& Gal-Petitfaux, 2009) qui ont confirmé des alternances dynamiques de " décrochages » (transgressions ludiques ou errance passive) - « raccrochages » (focalisation sur le travail scolaire) en relation à la situation vécue.

\subsubsection{Assumer le rôle d'arbitre et s'en détacher}

La première stratégie d'alternance montre que les élèves arbitres naviguent entre des préoccupations relatives au fait d'assumer le rôle d'arbitre confié par l'enseignant, et s'en détacher. Cette alternance est confirmée par les données motivationnelles. En effet, la motivation des élèves est principalement caractérisée par une régulation externe et une 


\section{eJRIEPS spécial 3 (janvier 2020)}

motivation intrinsèque à la connaissance et à l'accomplissement. Ces résultats peuvent être interprétés comme révélant chez les élèves: une envie de découvrir, mêlée à l'envie d'apprendre ce rôle social d'arbitre (motivation intrinsèque à la connaissance et à l'accomplissement) tout en répondant à des pressions sociales externes puisque ce rôle leur a été prescrit par l'enseignant (régulation externe) et qu'il supporte une visibilité publique au sein de la classe.

En d'autres termes, les élèves arbitres sont tiraillés entre les pressions sociales relatives à ce rôle (donnant lieu à une régulation externe) tout en souhaitant par ailleurs l'assumer puisqu'il offre la liberté de signaler ou pas une faute de jeu (en lien avec les motivations intrinsèques à la connaissance et à l'accomplissement).

Dans les moments du match où les élèves assument seuls leur rôle d'arbitre, ils cherchent à satisfaire prioritairement un besoin de compétence en mobilisant les connaissances nécessaires au rôle et en cohérence avec ce qu'implique la responsabilité de le tenir. Dans ce cas, l'élève arbitre prend acte de l'incertitude relative de ses choix et se confronte à l'obligation d'en assumer les conséquences. Il expérimente ainsi à la fois la liberté de choix et l'évaluation de ces choix par l'enseignant ou les autres élèves. En ce sens, l'arbitrage est bien une expérience de responsabilité à travers ces dimensions décisionnelles et évaluatives qui la caractérisent (Jaeger, 2009).

Toutefois, les pressions sociales et les dimensions décisionnelles et évaluatives de la responsabilité inhérentes à ce rôle conduisent parfois les élèves à s'en détacher. L'analyse des cours d'expérience des élèves a mis en évidence des similarités avec les arbitres experts quant aux raisons de ces détachements notamment en ce qui concerne le stress (Taylor \& Daniel, 1988). Les élèves arbitres ont tendance à « décrocher » lorsqu'ils éprouvent un stress lié au risque d'émergence de conflits interpersonnels et à la crainte de faire des erreurs arbitrales. Ils exploitent alors des opportunités offertes par certains temps faibles de jeu (après un but marqué ou lors d'une remise en jeu) pour faire une pause cognitive et émotionnelle quitte à laisser les joueurs gérer seul des moments du match. C'est le cas de Martin lorsqu'il se détache momentanément de son rôle d'arbitre lors d'une remise en jeu qu'il ne siffle pas. Cette « absence » de l'élève arbitre ne déclenche aucun conflit avec les joueurs qui réalisent, à leur initiative, sans intervention de l'élève arbitre, la remise en jeu. Dans ces moments où ils se délestent du rôle d'arbitre en laissant les joueurs s'auto-arbitrer, les élèves font l'expérience d'une responsabilité « déléguée » (Jourand et al., 2017). Cette expérience intègre alors une dimension coopérative de la responsabilité (Jardin, 2008) dans le sens où l'élève arbitre suppléait aux joueurs une part de son rôle. 


\section{eJRIEPS spécial 3 (janvier 2020)}

Dans d'autres cas, le décrochage est lié à des préoccupations étrangères au match. C'est le cas de Lucie lorsqu'elle rigole avec des camarades spectateurs. Dans ces moments rappelant les transgressions ludiques dans les leçons (Vors \& Gal-Petitfaux, 2009), l'élève arbitre ne cherche plus à satisfaire son besoin de compétence mais à se raccrocher à son statut d'élève dont il s'était éloigné de par son rôle. C'est bien le besoin d'affiliation qui est alors recherché, éloignant pour un temps l'élève de l'expérience de responsabilité dans la mesure où il ne fait plus de choix arbitraux et n'en assume plus les conséquences (JannerRaimondi, 2015).

Lorsqu'ils raccrochent, les élèves arbitres saisissent des opportunités qui sont signifiantes de leur point de vue dans la situation : par exemple, le fait de se trouver à proximité du ballon peut être perturbant pour eux et les amener à assumer de nouveau leur rôle.

\subsubsection{Arbitrer seul et s'aider des autres}

Cette deuxième stratégie d'alternance traduit un besoin d'autonomie des élèves inférieur à leur besoin de compétence (résultats issus des données motivationnelles). Les élèves arbitres n'éprouvent donc pas systématiquement le besoin de se détacher de l'enseignant pour prendre leurs décisions et en assumer les conséquences. A plusieurs reprises, ils saisissent l'opportunité que représente la présence d'élèves joueurs estimés plus compétents qu'eux ou de l'enseignant pour prendre leurs décisions. Ils sollicitent alors ces personnes ou se saisissent de leurs remarques concernant une faute, pour ensuite prendre eux-mêmes leur décision et l'assumer. En s'aidant ainsi des autres, ils satisfont leur besoin de compétence en tant qu'arbitre, même si cette compétence s'appuie sur celle des autres. Il semblerait alors que la responsabilité se construise d'abord sur la base d'un besoin de compétence alterné avec un besoin d'affiliation pour ne pas se sentir exclu du groupe classe. Ce besoin de compétence amènerait les élèves arbitres à s'aider des autres pour se sentir compétents dans leur rôle. Ce qui leur permettrait ensuite d'éprouver le besoin de se détacher des autres (élèves estimés plus compétents et enseignant) et donc de satisfaire leur besoin d'autonomie en assumant seul la responsabilité d'arbitrer.

Cette stratégie accrédite l'idée selon laquelle la classe forme un système éco-social, au sein duquel les agencements sociaux (des élèves occupant les rôles de joueurs ou de remplaçants), matériels (des bancs placés au bord du terrain de jeu pour y maintenir assises les équipes en attente), symboliques et culturels (l'enjeu d'un match, les idées et valeurs cristallisées dans le rôle d'arbitre) constituent des « affordance networks » (Adé et al., 2013 ; Barab \& Roth, 2006). Lors de la situation de match, l'élève arbitre tire bénéfice de tous les 


\section{eJRIEPS spécial 3 (janvier 2020)}

possibles de l'environnement de la leçon pour être efficace dans son rôle par l'entraide recherchée ou offerte par d'autres élèves ou l'enseignant. Qu'il s'agisse de saisir une offre d'aide ou d'en solliciter, ces aides se sont toujours déroulées entre l'élève arbitre et un autrui estimé par lui comme plus compétent ou légitime. C'est ainsi que Jules a saisi l'offre d'aide de Gautier reconnu par son statut de joueur de club. Ces offres et demandes d'aides sont fréquentes dans les leçons d'EPS (Adé et al., 2013 ; de Keukelaere, Guérin \& Saury, 2008 ; Huet \& Saury, 2011; Jourand et al., 2017; Saury et al., 2013). Elles participent à l'engagement durable des élèves dans les situations, à la viabilité des leçons et constituent un réseau de ressources pour les élèves vivant alors une expérience de "responsabilité partagée ». Cette responsabilité partagée traduit la dimension intersubjective de toute responsabilité (Jardin, 2008). De façon spéculative, nous pensons que la mise en visibilité particulière de l'activité de l'arbitre (seul sur le terrain à diriger le jeu au milieu des « joueurspairs », à la vue de l'enseignant et d'élèves en attente) stimulerait l'émergence des aides spontanées entre élèves car ils manifesteraient entre eux des attitudes solidaires voire empathiques vis-à-vis de l'élève en situation d'arbitrer participant à la construction de normes communes entre élèves au sein de la classe à propos de ce qu'il est admis de faire ensemble (Crance, Trohel, \& Saury, 2012 ; Saury et al., 2010). En effet, les élèves d'une classe partagent (ou non) un ensemble de normes, valeurs et pratiques légitimes, préfigurant notamment la façon de s'adapter aux situations scolaires (de Keukelaere et al., 2008 ; Saury \& Rossard, 2009 ; Saury et al., 2010) et notamment lorsqu'il s'agit d'occuper des responsabilités. Ces phénomènes, qui concrétisent des « micro-cultures » de classes, semblent aussi être tournés vers la préservation du statut d'élève pour celui occupant le rôle d'arbitre.

\subsection{Des stratégies d'intervention en EPS sur la base de gestalts}

Les deux stratégies d'alternance font émerger trois gestalts dans l'activité d'arbitrage des élèves. Le rapprochement qui s'opère ici avec la Gestalthéorie (Guillaume, 1937 ; Rosenthal \& Visetti, 1999) conduit à regarder l'activité des élèves arbitres comme une forme associée à un sens (Cizeron, 2013 ; Cizeron \& Ganière, 2016 ; Ganière, 2015) qui émerge en situation d'arbitrage. Comme forme, elle est repérable en situation de match, notamment par l'enseignant, en tant qu'elle possède des caractéristiques observables relatives à ce que les élèves donnent à voir. Comme sens, elle révèle l'expérience vécue par les élèves et les caractéristiques motivationnelles associées. 


\section{eJRIEPS spécial 3 (janvier 2020)}

\subsubsection{L'identification de trois gestalts}

Trois gestalts ont été identifiées. La première a été nommée « à proximité de la balle ». En termes d'observables, elle se repère lorsque l'élève arbitre reste près du ballon. En termes de sens, elle traduit la préoccupation de l'élève d' « assumer son rôle d'arbitre ». Pour être efficace, l'élève arbitre colle au ballon, ce dernier étant vécu comme cristallisant les fautes potentielles à repérer pour les siffler. Cette forme apparait chez des élèves qui sont principalement animés par une régulation externe ou une motivation intrinsèque à la connaissance ou à l'accomplissement lorsqu'ils occupent le rôle d'arbitre. Ils cherchent prioritairement à satisfaire leur besoin de compétence dans la situation en tentant seuls de faire respecter les règles. Cette forme "à proximité de la balle » correspond à des élèves arbitres animés principalement par une activité de « jugement de fait » (Rix, 2005), c'est-àdire sensibles à l'application stricte du règlement, au repérage de fautes à signaler dès leur survenue. Ces traits peuvent être associés à ceux caractérisant les « arbitres théoriciens " (Garncarzyk, 1995), centrés sur la dimension règlementaire. Cette gestalt traduit un élève arbitre assumant ses choix et actions en tant qu'il en est le véritable et le seul auteur (Jaeger, 2009).

La deuxième gestalt a été désignée comme une forme «à proximité d'autrui ». Elle se repère par un élève arbitre cherchant à se placer près de l'enseignant ou d'élèves pour y chercher de l'aide et satisfaire son besoin de compétence. Du point de vue du sens, elle renvoie à des élèves dont la préoccupation est de « s'aider des autres ". Elle traduit la volonté de satisfaire un besoin de compétence mais en partageant la responsabilité du rôle d'arbitre avec d'autres élèves estimés plus compétents ou avec l'autorité enseignante. Elle traduit une responsabilité partagée, c'est à dire une forme de responsabilité coopérative (Janner-Raimondi, 2015 ; Jardin, 2008 ; Piaget, 1932) dans laquelle les élèves s'offrent la possibilité de comparer leur point de vue avec celui d'autrui pour prendre leur propre décision. L'activité des élèves arbitres est alors comparable au « jugement délibéré " (Rix, 2005) des arbitres experts lorsqu'ils sollicitent de l'assistance auprès de pairs ou de la vidéo pour confirmer/infirmer une faute qu'ils pensent avoir repérée.

La troisième gestalt caractérise une forme « à distance ». Elle est reconnaissable lorsque des élèves arbitres sont positionnés à la périphérie du terrain, loin des joueurs et de l'action de jeu en cours, mais toujours proches de camarades en attente sur le bord du terrain. $\mathrm{Ce}$ placement est significatif de leur préoccupation de «se délester du rôle d'arbitre ». Par ce placement, certains élèves arbitre trouvent des occasions pour s'amuser avec des pairs et satisfaire leur besoin d'affiliation dont ils s'étaient écartés en occupant le rôle. En 


\section{eJRIEPS spécial 3 (janvier 2020)}

abandonnant ponctuellement leur responsabilité, ils sont assimilables aux arbitres "dilettantes » identifiés par Garncarzyk (1995). Pour d'autres, le sens de ce placement résulte de l'estimation qu'ils font de la capacité des joueurs à s'auto-arbitrer. Dans ces cas, cette forme «à distance » caractérise ce que nous avons qualifié de responsabilité " déléguée ».

Ces gestalts nous semblent être des analyseurs pertinents pour aider à l'intervention en EPS et engager les élèves dans le rôle d'arbitre de façon rassurante et potentiellement bénéfique pour les apprentissages notamment ceux liés à la responsabilité.

\subsubsection{Pistes possibles d'intervention}

Les trois gestalts identifiées peuvent être assimilées à des " artefacts » (Adé, 2018 ; GalPetiffaux \& Durand, 2001) pour l'enseignant dans la mesure où elles permettent d'amplifier son pouvoir d'action en l'aidant à mieux « lire » les conduites des élèves et le contexte dans lequel ces actions se dévoilent pour concevoir des dispositifs propices aux apprentissages. Ainsi nos résultats nous permettent de pointer (1) la difficulté pour les élèves à tenir le rôle, (2) la dimension inévitablement collective de l'activité d'arbitrage (par délégation ou recherche d'aide) et (3) des stratégies de placement (e.g., proche du ballon vs loin des actions de jeu) et de déplacement de l'élève arbitre (mobile dans l'aire de jeu vs statique en dehors des limites du terrain).

Les propositions que nous faisons visent alors à " trouver des solutions pour simplifier la complexité » (Berthoz, 2009, p. 11) que représente l'expérience de responsabilité de l'élève l'arbitre, telle qu'elle est visée par les programmes. Cette perspective de simplification de la complexité s'incarne dans le concept de simplexité (Berthoz, 2009), repris dans le domaine de la formation professionnelle (Durand, 2009 ; Durand, Ria, \& Veyrunes, 2010 ; Faïta \& Saujat, 2010) pour qualifier d'authentique un dispositif de formation mais dont la complexité y est réduite momentanément par élimination d'éléments contextuels estimés comme non essentiels par le formateur à cette étape de la formation, dans le but de faciliter l'accessibilité du dispositif de formation aux formés (Durand \& Salini, 2011).

Concernant la difficulté à tenir le rôle il s'agit par exemple pour l'enseignant de simplifier pour les élèves le rôle d'arbitre en ôtant ou en modifiant certains éléments mais en préservant un degré de complexité permettant de concevoir un contexte favorable aux apprentissages en lien avec les caractéristiques de la responsabilité. Cela pourrait donc permettre de renforcer les niveaux modérés de motivation intrinsèque à la connaissance et à l'accomplissement mis en évidence dans cette étude et de favoriser le développement 


\section{eJRIEPS spécial 3 (janvier 2020)}

d'une régulation identifiée plus importante chez les élèves. De façon plus explicite, une piste possible pour maintenir un élève en situation de responsabilité dans l'arbitrage est de lui offrir le choix, dès qu'il en éprouve le besoin, de saisir des occasions d'être assisté et ainsi de répondre à la dimension collective du rôle. Cette assistance peut être obtenue auprès d'un ou de deux élèves, choisis par l'élève arbitre sur la base d'une reconnaissance de leurs compétences dans l'arbitrage, dans la discipline sportive, ou dans leur capacité à rassurer et mettre en confiance. La recherche d'une assistance sera facilitée par l'aménagement de l'espace de la leçon. A titre d'illustration, on pourra délimiter, de chaque côté du terrain de jeu, un couloir à l'intérieur duquel l'arbitre assistant sera autorisé à se déplacer pour être sollicité. Cet aménagement pourrait participer à repositionner les élèves arbitre identifiés dans une forme « à distance » vers une forme "à proximité » de la balle tout en s'appuyant sur la forme "à proximité d'autrui ». Cependant, un excès d'assistance risquerait de privilégier une « responsabilité déléguée " au détriment d'une " responsabilité partagée » qui nous semble plus prometteuse en termes de responsabilisation de l'élève arbitre. Une alternative consisterait à limiter le nombre d'assistances possibles. Actualisée sous la forme d'une " assistance aidée », cette forme d'arbitrage s'assimile aux coopérations spontanées entre élèves identifiées dans nos résultats ainsi qu'à la prévalence des besoins de compétence et d'affiliation présente chez les élèves-arbitres.

D'autres formes d'aides sont possibles favorisant la réduction du caractère anxiogène et de la charge informationnelle qui caractérisent les difficultés du rôle en le distribuant à deux élèves arbitres évoluant dans l'aire de jeu. Plusieurs options sont alors envisageables. Une première consiste en une répartition spatiale limitant les déplacements et la part de l'arbitrage de chacun des arbitres à un demi-terrain. Cette option concentre chaque arbitre sur une partie du terrain et rend possible les opportunités de décrochage/raccrochage utiles aux élèves pour être efficaces. Une autre option vise à répartir entre les arbitres une partie des règles à faire respecter. Cet aménagement nous semble être une aide pour réduire la charge informationnelle, focaliser l'élève sur des dimensions du règlement, et proposer une expérience de responsabilité pouvant stimuler l'engagement du rôle d'arbitre et son apprentissage.

D'autres pistes pourraient être évoquées comme celle autorisant le ou les élèves arbitres à stopper le jeu pour consulter deux ou trois fois l'enseignant au cours d'un match. Cette proposition est convergente avec les trois préoccupations typiques mises en avant dans nos résultats et avec le niveau modérément faible dans le besoin d'autonomie des élèves- 


\section{eJRIEPS spécial 3 (janvier 2020)}

arbitres. Elle rappelle la possibilité offerte à certains arbitres professionnels de bénéficier de l'aide d'un assistant vidéo pour confirmer, infirmer, différer une décision en cas d'incertitude.

\section{Conclusion}

Cette étude poursuivait à la fois des ambitions méthodologiques et des visées épistémiques et transformatives. Du point de vue méthodologique, nous nous inscrivons dans la veine des travaux qui ambitionnent d'articuler des approches scientifiques différentes. Si nous avons fait le choix de combiner des données motivationnelles et d'expérience, nous encourageons l'investigation de notre objet d'étude avec d'autres options, comme par exemple celles proposées par les approches sociologiques et didactiques (Poggi \& BrièreGuenoun, 2014 ; Poggi \& Moniotte, 2011).

Du point de vue épistémique, notre étude permet de mettre en avant des stratégies d'élèves occupant le rôle social d'arbitre pour lequel ils sont souvent sollicités dans les leçons d'EPS mais qui reste pourtant peu investigué par la recherche. De ces stratégies ont été identifiées des gestalts qui nous semblent utiles pour des visées transformatives. Ainsi en pointant les liens possibles et heuristiques entre la recherche et l'activité professionnelle des enseignants nous défendons l'importance de prendre appui sur l'activité des élèves analysée in situ, afin d'aider les enseignants à concevoir des environnements d'apprentissage envisagés comme des espaces d'actions encouragées (Saury et al., 2013). Ainsi, en comprenant mieux l'expérience de responsabilité des élèves en tant qu'arbitres, les enseignants pourront mieux les former (Goigoux, Ria, \& Toczek-Capelle, 2010). Pour cela, étudier la dynamique d'apparition de ces gestalts en situation d'arbitrage semblerait judicieux. L'idée serait de voir si certaines de ces formes pourraient caractériser différents états dans la dynamique du processus de responsabilisation. De manière spéculative, nous pourrions supposer que la responsabilité « individuelle " s'incarnant dans la forme "à proximité du ballon » et traduisant la préoccupation d'assumer son rôle seul, traduirait un niveau supérieur de responsabilité par rapport à la forme "partagée » dans laquelle les élèves ressentent le besoin de "s'aider des autres " pour assumer leur rôle. Toutefois, nous pourrions envisager l'émergence, au cours de ce processus, d'états transitoires combinant des formes de responsabilité " partagée » et « individuelle ». II serait alors possible de définir une morphogénèse (Lesne \& Bourgine, 2006 ; Ganière, 2010 ; Cizeron, 2013 ; Cizeron \& Ganière, 2016) de la responsabilité en EPS à travers le rôle d'arbitre pour caractériser un parcours de formation « centré responsabilité ». 


\section{eJRIEPS spécial 3 (janvier 2020)}

\section{Bibliographie}

Adé, D. (2016). L'intervention « par » les objets matériels en EPS. Un éclairage à partir du programme de recherche du Cours d'action. Recherches \& éducations, 15, 107-120.

Adé, D. (2018). Expérience et matérialité dans les situations d'intervention en Éducation Physique et Sportive et d'expertise en sport. Contribution au programme de recherche scientifique et technologique du Cours d'action. Note de synthèse en vue d'une habilitation à diriger des recherches. Spécialité STAPS. Université de Rouen Normandie, Rouen, France.

Adé, D., Ganière, C., \& Louvet, B. (2018). The Role of the Referee in Physical Education Lessons: Student Experience and Motivation. Physical Education and Sport Pedagogy, 23(4), 1-13.

Adé, D., Picard, M., \& Saury, J. (2013). Les ressources exploitées par les élèves pour agir dans un format pédagogique en atelier: Une étude empirique lors de leçons de musculation en Education Physique et Sportive. eJRIEPS, 30, 26-50.

Allen, J. D. (1986). Classroom management: Student's perspectives, goals and strategies. American Educational Research Journal, 23, 437-459.

Barab, S. A., \& Roth, W. M. (2006). Curriculum-based ecosystems: Supporting knowing from an ecological perspective. Educational Researcher, 35(5), 3-13.

Berthoz, A. (2009). La simplexité. Paris: Odile Jacob.

Boiché, J., Sarrazin, P., Grouzet, M., Pelletier, L., \& Chanal, J. (2008). Students' motivational profiles and achievement outcomes in physical education: a self-determination perspective. Journal of Educational Psychology, 3, 688-701.

Cizeron, M., \& Ganière, C. (2016). Élaborer des connaissances utiles aux enseignants pour guider les apprentissages des élèves en EPS : l'étude des morphogenèses des habiletés motrices. eJRIEPS, 38, 133-168.

Cizeron, M. (2013). Du savoir à l'activité : la supervision active et le guidage des apprentissages en éducation physique et en sport. Note de synthèse en vue de l'obtention d'une habilitation à diriger des recherches, Spécialité STAPS, Université Blaise Pascal, Clermont-Ferrand II, France.

Crance, M. C., Trohel, J., \& Saury, J. (2012). The experience of a highly skilled student during handball lessons in Physical education: a relevant pointer to the gap between school and sports contexts of practice. Physical Education \& Sport Pedagogy, 18, 103-115. 


\section{eJRIEPS spécial 3 (janvier 2020)}

Cury, F., Famose, J-P., \& Sarrazin, P. (1994). Mesurer différents aspects de la motivation d'accomplissement en sport: propriétés psychométriques de l'IMI, de l'EPCM, et du POSQ. Intervention au Congrès International de la SFPS, Poitiers, France.

De Keukelaere, C., Guérin, J., \& Saury, J. (2008). Co-construction de connaissances chez les élèves en EPS au cours d'une situation d'apprentissage en volley-ball. STAPS, 79(1), 23-38.

Deci, E., \& Ryan, R. (2002). Handbook of self-determination research. Rochester, NY: University of Rochester Press.

Dosseville, F., Laborde, S., \& Raab, M. (2011). Contextual and personal motor experience effects in judo referees' decisions. Sport Psychologist, 25, 67-81.

Durand, M., \& Salini, D. (2011). Incorporation, parcimonie et élégance de l'expérience au travail : vers une «simplexification» des formations professionnelles. Travail et Apprentissages, 7, 81-93.

Durand, M. (2009). La conception d'environnement de formation sous le postulat de l'énaction. In M. Durand, et L. Filliettaz (Eds.). Travail et formation des adultes (pp. 191-225). Paris: PUF.

Durand, M. (2014). Activité humaine, pratiques sociales et éducation des adultes. In J. Friedrich, et J. Pita Castro (Eds.), Recherche en formation des adultes: Un dialogue entre concepts et réalité, (pp.13-39). Dijon : Editions Raison \& Passions.

Durand, M., Ria, L., \& Veyrunes, P. (2010). Analyse du travail et technologie de formation : signification et organisation de l'activité des enseignants. In F. Saussez, F. Yvon, et F. Loyola (Eds.), Analyser l'activité enseignante : des outils méthodologiques et théoriques pour l'intervention et la formation (pp. 17-39). Québec : Presses de l'Université de Laval.

Faïta, D., \& Saujat, F. (2010). Développer l'activité des enseignants pour comprendre et transformer leur travail : un cadre théorique et méthodologique. In F. Yvon, et F. Saussez (Eds.), Des outils théoriques et méthodologiques pour l'intervention et la formation (pp. 41-69). Laval: Presses Universitaires de Laval.

Gal-Petitfaux, N., \& Durand, M. (2001). L'enseignement de l'Education physique comme «action située » : proposition pour une approche d'anthropologie cognitive. STAPS, $55,79-100$.

Ganière, C. (2010). Morphogénèse du saut de mains : croisement de données objectives (biomécaniques, cinématiques et dynamiques) et de l'expérience subjective du 


\section{eJRIEPS spécial 3 (janvier 2020)}

gymnaste. Mémoire de Master I. Université Blaise Pascal, Clermont-Ferrand II, France.

Ganière, C. (2015). L'organisation motrice de l'élève apprenant en EPS : Elaboration d'un cadre d'analyse pour l'intervention. Thèse de doctorat non publiée, Université Blaise Pascal, Clermont-Ferrand II, France.

Garncarzyk, C. (1995). Représentations de l'arbitrage : Consensus ou divergences ? Science et Motricité, 26, 9-20.

Goigoux, R., Ria, L., \&, Toczek-Capelle, M-C. (2010). Mieux connaître les parcours de formation des enseignants débutants pour mieux les former. In R, Goigoux, L, Ria, et M-C, Toczek-Capelle (Eds.), Les parcours de formation des enseignants débutants (pp.25-44). Clermont-Ferrand : Presses universitaires de l'Université Blaise Pascal. Guillaume, P. (1937). La psychologie de la forme. Paris : Flammarion Hastie, P., \& Siedentop, D. (1999). An ecological perspective on physical education. European Physical Education Review, 5(1), 9-29.

Hastie, P., \& Siedentop, D. (2006). The classroom ecology paradigm. In D. Kirk, D. Macdonald, and M. O'Sullivan (Eds.), The handbook of physical education (pp. 214225). London: Sage.

Huet, B., \& Saury, J. (2011). Ressources distribuées et interactions entre les élèves au sein d'un groupe d'apprentissage : une étude de cas en éducation physique et sportive. eJRIEPS, 24, 4-30.

Jaeger, M. (2009). Du principe de responsabilité au processus de responsabilisation. Vie sociale, 3(3), 71-81.

Janner-Raimondi, M. (2015). Formes et pratiques de conseils d'élèves: quelle(s) responsabilité(s) en jeu ? Les sciences de l'éducation. Pour l'Ere nouvelle, 48(1), 2345.

Jardin, E. (2008). Soyez réaliste, demandez l'impossible. Partie 2 : Mai 68 : un problème en héritage. Les Sciences de l'éducation - Pour l'Ére nouvelle, 44(3), 73-90.

Jenkinson, K. A., Naughton, G., \& Benson, A. C. (2014). Peer-assisted learning in school physical education, sport and physical activity programs: a systematic review. Physical Education and Sport Pedagogy, 19(3), 253-277.

Jourand, C., Adé, D., Sève, C., Komar, J., \& Thouvarecq, R. (2017). Dynamics of Student Interactions: an Empirical Study of Orienteering Lessons in Physical Education. Physical Education \& Sport Pedagogy, 23(2), 134-149. 


\section{eJRIEPS spécial 3 (janvier 2020)}

Lafont, L. (2012). Cooperative learning and tutoring in sports and physical activities. In A. Casey (Ed.), Cooperative learning in Physical Education: A research basic approach (pp. 136-149). Oxon: Routledge.

Lafont, L., Rivière, C., Darnis, F., \& Legrain, P. (2016). How to structure group work? Conditions of efficacy and methodological considerations in physical education. European Physical Education Review, 13, 1-12.

Lerbet, G. (1992). L'école du dedans. Paris : Hachette.

Lesne, A., \& Bourgine, P. (2006). Introduction. In P. Bourgine, et A. Lesne (Eds.), Morphogénèse. L'origine des formes (pp. 13-26). Paris : Belin.

Louvet, B. (2011). Jaune, rouge... Les hommes en noirs en voient-ils de toutes les couleurs ? Analyse psychologique d'une activité stressante. Dans F. Dosseville, et C. Garncarzyck (Eds.), Les facettes de l'arbitrage : Problématiques et recherches actuelles. Paris : Publibook.

Maturana, H., \& Varela, F. (1994). L'arbre de la connaissance: Racines biologiques de la compréhension humaine. Paris: Editions Addison-Wesley France.

McAuley, E., Duncan, T., \& Tammen, V. (1989). Psychometric properties of the Intrinsic Motivation Inventory in a competitive sport setting : a confirmatory factor analysis. Research Quarterly for Exercice and Sport, 60, 48-58.

Ministère de l'Education Nationale. (2010). BO n4 du 29 avril 2010.

Ministère de l'Education Nationale. (2015). BO n॰11 du 26 novembre 2015.

Ministère de l'Education Nationale. (2019). BO n`1 du 22 janvier 2019.

Piaget J. (1932). Le jugement moral chez l'enfant. Paris : PUF.

Poggi, M-P., \& Brière-Guenoun, F. (2014). Ce qui s'enseigne en milieu difficile : tentative d'articulation des approches sociologique et didactique. In N. Wallian, M.-P. Poggi, et A. Chauvin-Vileno (Eds.), Action, interaction, intervention : à la croisée du langage, de la pratique et des savoirs (pp. 333-364). Berne : Peter Lang.

Poggi, M-P., \& Moniotte, J. (2011). Quelle place pour le sociologique dans les recherches en didactique de l'EPS ? Éducation et didactique, 5 (1), 29-44.

Rix, G. (2005). Typologie des actes de jugement de l'arbitre de rugby expérimenté. Movement \& Sport Sciences, 56, 109-124.

Rix-Lièvre, G., Boyer, S., Coutarel, F., \& Lièvre, P. (2014). La performance arbitrale : de son étude à son développement ", Activités [En ligne], 11-1 | Avril 2014, URL : http://activites.revues.org/425.

Rosenthal, V., \& Visetti, Y. M. (1999). Sens et temps de la gestalt. Intellectica, 28, 147-227. 


\section{eJRIEPS spécial 3 (janvier 2020)}

Saury, J., \& Rossard, C. (2009). Les préoccupations des élèves durant des tâches d'apprentissage coopératives et compétitives en badminton : une étude de cas. Revue des Sciences de l'Education, 35(3), 195-216.

Saury, J., Adé, D., Gal-Petitfaux, N., Huet, B., Sève, C., \& Trohel, J. (2013). Actions, significations et apprentissages en EPS. Paris : Editions. EP.S.

Saury, J., Huet, B., Rossard, C., \& Sève, C. (2010). Dispositifs de co-observation et configurations d'activités en éducation physique et sportive. In I. de Saint-Georges, et D. Adé (Eds.), Les objets dans la formation (pp. 143-159). Toulouse : Octarès.

Sève, C., \& Terré, N. (2018). L’EPS du dedans. Dossier EPS n84. Paris : Editions Revue EP.S.

Sève, C., \& Saury, J. (2010). Un programme de recherche en STAPS fondé sur la théorie du cours d'action. eJRIEPS, 20, 93-108.

Taylor, A. H., \& Daniel, J. V. (1988). Sources of stress in soccer officiating : An empirical study. In T. Reilly, A. E. Lees, K. Davids, and W. J., Murphy (Eds.), Science and Football : Proceedings of the First World Congress of Science and Football (pp.538544). London : E. \& F. N. Spon.

Theureau, J. (2002). La description du cours d'action en termes de signes hexadiques et ses options philosophiques. Communication présentée à l'Exploration de l'expérience et pratique phénoménologique (IV) : qu'est-ce que décrire, Paris. France.

Theureau, J. (2004). Le cours d'action. Méthode élémentaire. Toulouse : Octarès.

Theureau, J. (2006). Le cours d'action : méthode développée. Toulouse : Octarès.

Vallerand, R. J. (1997). Toward a hierarchical model of intrinsic and extrinsic motivation. In M. P. Zanna (Ed.), Advances in experimental social psychology (pp. 271-360). NewYork: Academic Press.

Varela, F. J. (1989). Autonomie et connaissance. Essai sur le vivant. Paris : Seuil.

Vors, O., \& Gal-Petitfaux, N. (2009). Construire une activité collective de travail dans une classe d'EPS en «Réseau ambition réussite »: entre masquage et ostentation. eJRIEPS, 18, 156-177. 